\title{
Detecting Incipient Radial Deformations of Power Transformer Windings using Polar plot and Digital Image Processing
}

\author{
A. Abu-Siada ${ }^{1 *}$, O. Aljohani ${ }^{1}$ \\ ${ }^{1}$ Electrical and Computer Engineering Department, Curtin University, Perth, Western Australia \\ *a.abusiada@curtin.edu.au
}

\begin{abstract}
One of the main drawbacks of the frequency response analysis (FRA) technique that is widely accepted as the most reliable tool to detect transformer internal mechanical deformations is the inconsistent interpretation of the measured signature because of its reliance on personal expertise more than standard codes. Moreover, conventional FRA signature has a very low accuracy in detecting incipient and low mechanical fault levels. In order to avoid inconsistent interpretation for the transformer FRA signatures and improve its accuracy to detect minor fault levels, a reliable automated technique has become essential. This paper investigates the feasibility of utilizing FRA polar plot to detect minor radial deformation levels within two, 3-phase power transformers of different ratings and winding configurations simulated using three-dimensional finite element analysis software. Simulation results are validated through experimental measurements. Results of this paper are also compared with the results obtained for other types of transformer winding deformations that are published in the literature in order to identify unique impact for each fault type on the proposed method. Findings reveal the superiority of the proposed approach over existing conventional technique in terms of accurate identification and quantification for minor transformer winding deformations.
\end{abstract}

\section{Introduction}

Condition monitoring and diagnosis techniques have become essential to maintain the reliability of various assets within electrical power transmission and distribution networks. As power transformer represents a key asset in such networks, it has been given much attention through regular monitoring of its health condition to detect any incipient faults as early as possible [1-3]. In case of minor mechanical winding deformation that may occur as a result of short circuit faults, transformer may continue work normally however; transformer mechanical integrity is significantly deteriorated [3]. Among the several techniques currently used to evaluate the condition of the transformer, frequency response analysis (FRA) has been considered as the most reliable tool to detect transformer mechanical integrity [4]. Owing to the fact that power transformer can be modelled as electrical equivalent circuit comprising capacitive and inductive elements, magnitudes of these electrical circuit parameters are changing as a result of any physical deformation within the transformer windings and hence changing the transformer frequency response characteristic that can be measured using frequency response analyser. This device injects a low voltage of variable frequency to each phase of the off-service transformer and measures the response signal at the other terminal of the same phase [3,5]. While measurement method is well developed and standardized, analysis of the measured FRA signature is not a straight forward process and requires high level of professional expertise. As a result, different conclusions may be reported for the same FRA signature. Several papers investigating the impacts of various winding deformations on the transformer FRA signature can be found in the literatures [5-7]. However, similar to current industry practice, most of the published studies have only focused on the magnitude of the measured
FRA signature without considering the phase angle plot. A few studies in the literature introduced some statistical indices such as standard deviation (SD), coefficient of correlation (CC), and absolute sum of logarithmic error (ASLE) that can be calculated using the numerical values (raw data) of the FRA magnitude signature to ease the interpretation process $[8,9]$. However, these statistical parameters were reported to be inadequate tools for quantifying and identifying the investigated signatures [9]. The SD index is dominated by the data scattered around the high magnitudes of the FRA signature which may lead to a wrong conclusion $[10,11]$. The $\mathrm{CC}$ fails to calculate the correct correlation between two signatures under certain circumstances [12]. On the other hand, the ASLE was reported as an immature indicator that needs further investigations [13]. Recently, an attempt to utilize FRA polar plot along with digital image processing (DIP) techniques in order to standardize and automate the FRA interpretation process was introduced [14-16]. While the feasibility of this new approach was investigated to detect winding shorted turns [14], axial displacement and disk space variation [15], bushing faults and insulating oil deterioration [16], it has not been investigated to detect radial faults yet. As such, the main contribution of this paper is:

-investigating the feasibility of utilizing FRA polar plot signature along with DIP techniques to detect minor levels of transformer winding radial deformations.

-providing a detailed comparison of the results obtained for various types of winding deformations to identify unique threshold levels for each fault type and level.

In this context, two power transformer models of different ratings, winding structures and sizes are simulated using $3 \mathrm{D}$ finite element analysis and minor levels of windings radial deformations at different locations are implemented to investigate their impacts on the proposed polar plot. 
Moreover, practical measurements have been conducted on a single-phase transformer to validate simulation results. (a)

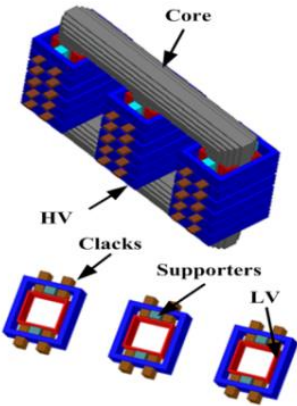

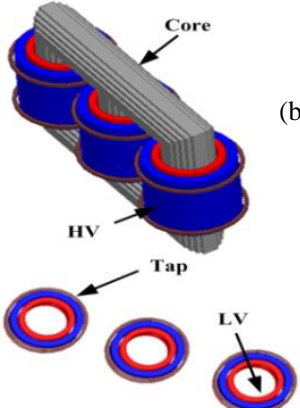

(b)

Fig. 1. 3D transformer models: (a) 10kVA and (b) 40MVA (b)
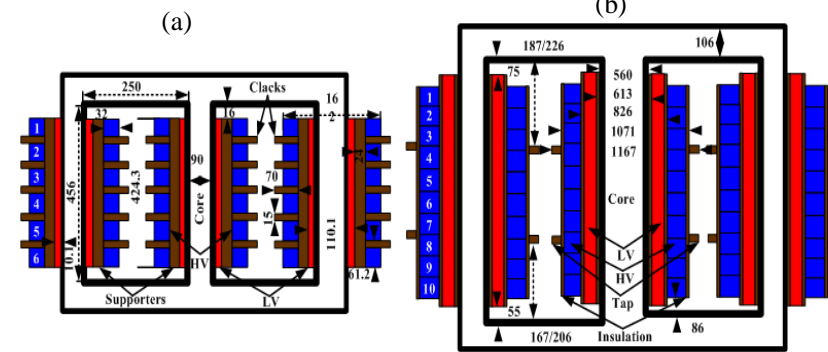

Fig. 2. Dimensions (in mm): (a) 10kVA and, (b) 40MVA
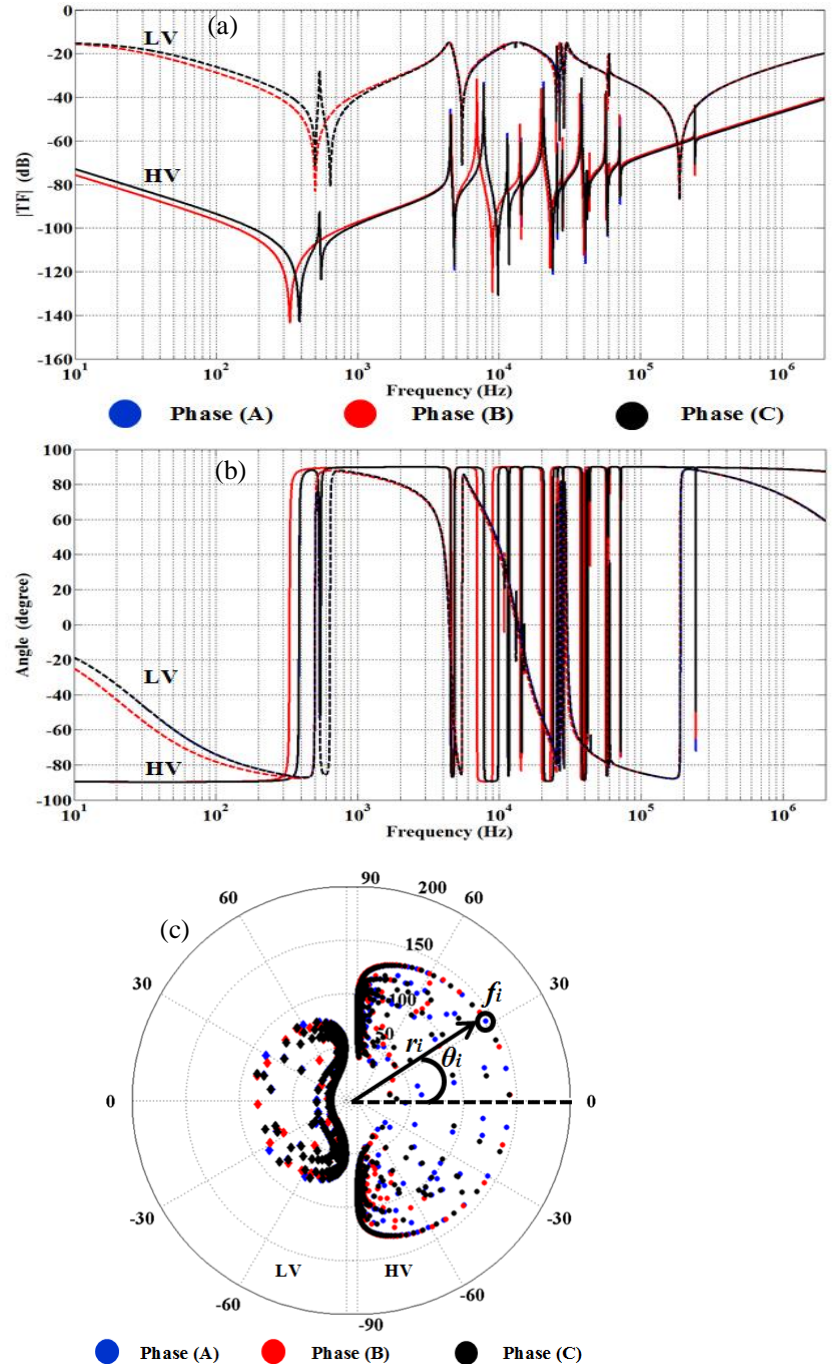

Fig. 3. 10kVA transformer reference FRA signature (a) magnitude, $(b)$ angle and (c) polar plot

\section{Finite element analysis}

Finite element analysis (FEA) is a computer-based technique that is widely used to emulate the performance of real systems [17]. It can effectively analyse the magnetic and electric fields within power transformers under different operating conditions [18]. Three-dimensional (3D) models of two power transformers rated at $10 \mathrm{kVA}$ and $40 \mathrm{MVA}$ as shown in Fig. 1 are simulated using FEA based on their physical dimensions (Fig. 2). The low voltage (LV) windings within the 10kVA transformer are made of a continuous layer comprising 140 turns in each phase while the high voltage (HV) windings consist of six disks with 1134 turns. On the other hand, the windings of the 40MVA transformer are of circular configuration in which the continuous layer of the LV winding comprising 200 turns and the HV winding is made of 10 disks with 120 turns per disk. The detailed specifications of the two transformers can be found in [14-16]. The parameters of the transformer equivalent electric circuit are calculated using Ansoft Maxwell application as explained in $[19,20]$.

\section{Proposed polar plot signature}

FEA technique is used to plot the FRA signature of each phase of the LV and HV windings within the 10kVA and 40MVA transformers. The FRA signature is obtained through injecting a sweep frequency, 10 volts AC voltage $\left(V_{\text {in }}\right)$ to each phase and measuring the response signal $\left(V_{\text {out }}\right)$ at the other terminal of the same phase. The measured healthy (reference) FRA signature is then plotted as magnitude and phase angle of the transfer function $\left(\mathrm{TF}=V_{\text {out }} / V_{\text {in }}\right.$ in $\left.\mathrm{DB}\right)$. Fig. 3(a) and (b) show the conventional reference FRA signature of the $10 \mathrm{kVA}$ transformer. The FRA signature of the 40MVA transformer is similar to Fig. 3 with different magnitudes and resonance frequencies. Any variation in the FRA signature when compared with the reference signature is an indication for a fault. While FRA signature comprises magnitude and phase angle plots, only magnitude plot is conventionally used to diagnose transformer winding deformation. This affects the detection accuracy of the current FRA industry practice especially its ability to detect incipient mechanical faults. Moreover, as the current analysis technique relies on visual inspection along with some statistical coefficients as illustrated in the previous section, the conclusion for the same FRA signature is not always unique and different results may be reported by various experts. To enhance the FRA accuracy and automate the analysis process, the magnitude and phase angle plots are integrated in one polar plot as shown in Fig. 3 (c). In this plot, each scattered point involves 3 information: magnitude $\boldsymbol{r}$, phase angle $\theta$ at a particular frequency $f$ and hence it comprises more image features than the magnitude plot [14-16].

\section{Digital image processing techniques}

DIP techniques are computerized and electronic algorithms utilized to improve and automate the interpretation of pictorial information [21]. A digital image $D$ can be considered as a two-dimensional matrix of pixels. Each pixel located at $(i, j)$ in $x-y$ plan is represented by an image intensity $|d|$ [22]. A digital image processing code has been developed to extract some unique features from the polar plot image such as geometrical dimensions, invariant 
moments and texture analysis [22, 23]. The developed DIP code is illustrated in the flow chart of Fig. 4. The technique starts by acquiring the FRA polar plot signature from the frequency response analyser. The polar plot signature is then pre-processed to adjust the dimension, colour format and extension type after which it will be manipulated through segmentation process to identify signature boundaries and isolate noises. Geometric, invariant moments and texture features are then extracted from the processed image [24]. These features are utilized to calculate three classification parameters: city-block distance (CBD), root mean square (RMS) and image Euclidean distance (IED) [25-27], that are compared with the respective values of the reference signature for fault identification and quantification. The details of extracted features along with the three classification parameters can be found in [14-16] and hence they are not discussed here.

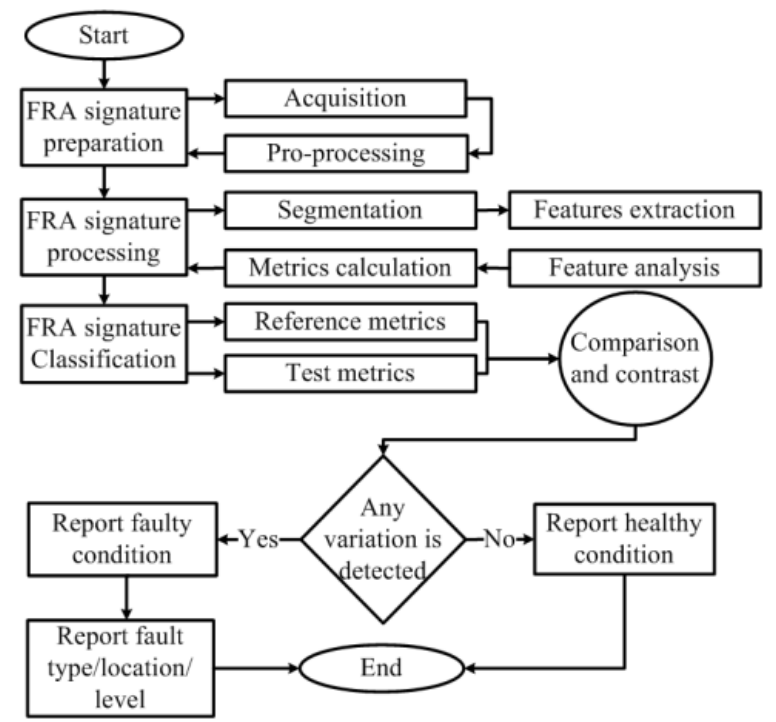

Fig. 4. Flow chart of the developed DIP code

Table 1 Reference geometric dimensions features for the investigated transformers

\begin{tabular}{c|c|c|c|c}
\hline \multirow{2}{*}{ Feature } & \multicolumn{2}{|c|}{$10 \mathrm{kV}$ A transformer } & \multicolumn{2}{c}{ 40MVA transformer } \\
\cline { 2 - 5 } & HV & LV & HV & LV \\
\hline$g_{1}$ & 30443 & 9600 & 66091 & 47692 \\
$g_{2}$ & 878.70 & 493.15 & 1837.44 & 1473.07 \\
$g_{3}(X)$ & 358.85 & 339.95 & 673.65 & 673.10 \\
$g_{3}(Y)$ & 289.88 & 289.91 & 612.83 & 560.22 \\
$g_{4}($ Major $)$ & 374.85 & 221.58 & 834.53 & 642.54 \\
$g_{4}$ (Minor $)$ & 109.41 & 59.24 & 199.37 & 127.23 \\
\hline
\end{tabular}

Table 2 Reference Invariant and Texture features for the investigated transformers

\begin{tabular}{|c|c|c|c|c|c|}
\hline \multirow{2}{*}{\multicolumn{2}{|c|}{ Feature }} & \multicolumn{2}{|c|}{$10 \mathrm{kVA}$ transformer } & \multicolumn{2}{|c|}{ 40MVA transformer } \\
\hline & & $\mathrm{HV}$ & LV & $\mathrm{HV}$ & LV \\
\hline 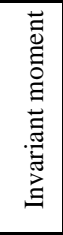 & $\begin{array}{l}\Phi_{1} \\
\Phi_{2} \\
\Phi_{3} \\
\Phi_{4} \\
\Phi_{5} \\
\Phi_{6} \\
\Phi_{7}\end{array}$ & $\begin{array}{c}0.2929 \\
0.0000 \\
0.0016 \\
-0.0054 \\
0.6358 \\
0.0975 \\
0.5423\end{array}$ & $\begin{array}{c}0.4745 \\
0.0000 \\
0.0017 \\
-0.0077 \\
1.7363 \\
0.4392 \\
1.4437\end{array}$ & $\begin{array}{c}0.6207 \\
0.0000 \\
0.0070 \\
-0.0320 \\
3.1864 \\
1.0943 \\
2.5769\end{array}$ & $\begin{array}{c}0.8109 \\
0.0000 \\
0.0072 \\
-0.0377 \\
5.2993 \\
2.3430 \\
4.3172\end{array}$ \\
\hline 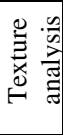 & $\begin{array}{l}\tau_{1} \\
\tau_{2} \\
\tau_{3} \\
\tau_{4}\end{array}$ & $\begin{array}{l}0.0779 \\
1.9611 \\
1.8222 \\
0.4876\end{array}$ & $\begin{array}{l}0.0611 \\
1.9694 \\
1.8588 \\
0.5075\end{array}$ & $\begin{array}{l}0.0573 \\
1.9714 \\
1.8664 \\
0.5295\end{array}$ & $\begin{array}{l}0.0521 \\
1.9740 \\
1.8789 \\
0.5301\end{array}$ \\
\hline & & 0.863598 & 1.091791 & 1.538326 & 2.345671 \\
\hline
\end{tabular}

The 15 extracted image features (4 geometric features, 7 invariant moments and 4 texture features) for the two investigated transformers with no deformed windings are listed in Tables 1 and 2, respectively. These features are to be stored for future comparison with future image features of the same transformer. The extracted geometric features (Table 1) indicate that the LV windings features are less than that of the HV windings. On the other hand, Table 2 shows the invariant moments and texture features of the $\mathrm{LV}$ windings are higher than that of the $\mathrm{HV}$ windings.

\section{Radial winding deformation detection}

The interaction of short circuit currents and magnetic flux can result in electromagnetic forces that may cause radial deformation to the transformer windings [3]. Due to these forces, the HV windings will be subjected to free radial buckling (pushed outward to the tank) whereas the inner winding will be subjected to compressive stress (forced radial buckling) that pushes the winding toward the core [20]. Consequently, forced radial buckling bends the LV windings in an alternative span while free buckling causes swell at one or more edge of the conductor. FEA is used to simulate such deformations on the two investigated transformers as shown in Fig. 5.

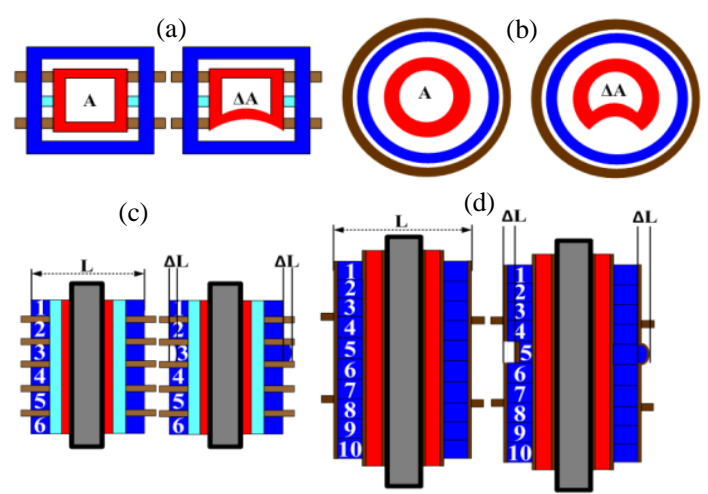

Fig. 5. Top view of healthy and forced radial buckling on the LV winding: (a) 10kVA, (b) 40MVA and front view of healthy and free radial buckling on the $H V$ winding: (c) lokVA (disk 3) and (d) 40MVA (disk 5)

The buckling fault level is calculated as below:

Forced buckling $=\frac{\text { area of deformed section }(\Delta A)}{\text { healthy cross section area }(A)} \times 100 \%$
Free radial buckling $=\frac{\text { Radial length }(\Delta L)}{\text { disk length }(L)} \times 100 \%$

\subsection{Case study 1: 10kVA transformer}

\subsubsection{Forced radial buckling on the $L V$ winding}

Six fault levels ( $1 \%$ to $5 \%$ and $40 \%$ ) of forced radial buckling within phase-A of the 10kVA transformer LV windings are simulated using FEA. The impact of these fault levels on conventional FRA signature (magnitude of the winding transfer function) is shown in Fig. 6(a). Results show that, except for the $40 \%$ fault level, all other minor fault levels are hard to detect using conventional FRA signature which is considered as one of the weaknesses of the conventional FRA interpretation approach. Transformer incipient mechanical faults should be identified as early as it emerges due to the progressive nature of such faults to avoid any potential catastrophic consequences. 


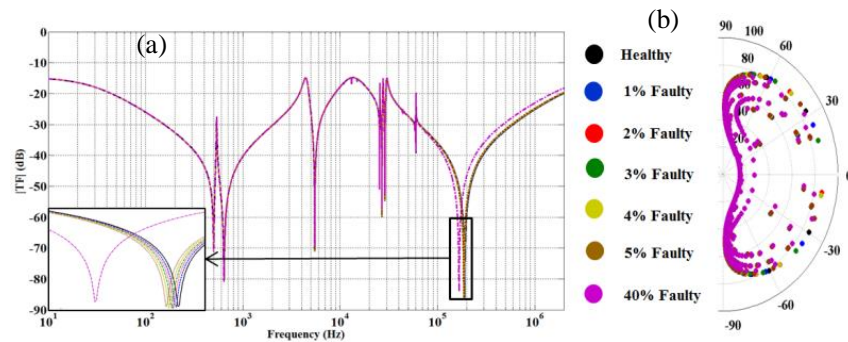

Fig. 6. Impact of $L V$ winding forced radial buckling fault on: (a) conventional approach and (b) polar plot approach

To overcome this substantial drawback in the conventional FRA signature, polar plots for all investigated forced radial buckling levels are obtained as shown in Fig. 6 (b). As mentioned above, the polar plot comprises more image features than the magnitude plot and facilitates the utilization of DIP techniques, which aids in standardizing and automating the FRA interpretation process. The developed DIP code is utilized to extract the proposed 15 image features from the polar plot images as listed in Table 3. Similar to the method in [13-15], geometric features are used to calculate the CBD metric (Fig. 7(a)) while RMS (Fig. 7(b)) and IED (Fig. 7(c)) are calculated based on the remaining 11 features. As can be seen in Fig. 7 (a-c), CBD, RMS and IED values are increasing consistently with the increase in fault level.

Table 3 The 15 extracted features of 10kVA transformer LV winding forced buckling

\begin{tabular}{c|c|c|c|c|c|c}
\hline \multirow{2}{*}{ Feature } & \multicolumn{6}{|c}{ Fault level } \\
\cline { 2 - 7 } & $1 \%$ & $2 \%$ & $3 \%$ & $4 \%$ & $5 \%$ & $40 \%$ \\
\hline$g_{1}$ & 9387 & 9362 & 9359 & 9344 & 9336 & 8793 \\
$g_{2}$ & 486.82 & 486.43 & 486.11 & 485.79 & 485.63 & 472.73 \\
$g_{3}(\mathrm{X})$ & 338.86 & 338.59 & 338.52 & 338.47 & 338.28 & 337.12 \\
$g_{3}(\mathrm{Y})$ & 288.96 & 288.78 & 288.57 & 288.44 & 288.38 & 288.07 \\
$g_{4}($ Maj. $)$ & 220.18 & 219.54 & 218.63 & 217.82 & 216.91 & 214.21 \\
$g_{4}($ Min. $)$ & 59.22 & 59.21 & 59.19 & 59.18 & 59.16 & 58.44 \\
\hline CBD & 222.8 & 249.29 & 253.82 & 270.14 & 279.48 & 617.47 \\
\hline$\Phi_{1}$ & 0.4751 & 0.4754 & 0.4758 & 0.4761 & 0.4767 & 0.4801 \\
$\Phi_{2}$ & 0.0000 & 0.0000 & 0.0000 & 0.0000 & 0.0000 & 0.0000 \\
$\Phi_{3}$ & 0.0016 & 0.0014 & 0.0013 & 0.0012 & 0.0011 & 0.0008 \\
$\Phi_{4}$ & -0.0074 & -0.0071 & -0.0067 & -0.0061 & -0.0058 & -0.0033 \\
$\Phi_{5}$ & 1.7428 & 1.7431 & 1.7438 & 1.7452 & 1.7464 & 1.7892 \\
$\Phi_{6}$ & 0.4427 & 0.4438 & 0.4453 & 0.4466 & 0.4482 & 0.4816 \\
$\Phi_{7}$ & 1.4322 & 1.4317 & 1.4311 & 1.4309 & 1.4305 & 1.4293 \\
\hline$\tau_{1}$ & 0.0605 & 0.0601 & 0.0597 & 0.0592 & 0.0586 & 0.0580 \\
$\tau_{2}$ & 1.9725 & 1.9736 & 1.9757 & 1.9778 & 1.9789 & 1.9806 \\
$\tau_{3}$ & 1.8627 & 1.8633 & 1.8667 & 1.8679 & 1.8681 & 1.8723 \\
$\tau_{4}$ & 0.5086 & 0.5094 & 0.5103 & 0.5118 & 0.5129 & 0.5174 \\
\hline RMS & 1.092663 & 1.093005 & 1.094014 & 1.094845 & 1.095310 & 1.103955 \\
\hline IED & 0.014615 & 0.015973 & 0.019156 & 0.021878 & 0.024046 & 0.026640 \\
\hline & & & & & & \\
\hline
\end{tabular}

\subsubsection{Free radial buckling on the $H V$ winding}

Various free radial buckling levels (1\% to $5 \%$ and $40 \%$ ) are simulated on phase-A of the $10 \mathrm{kVA}$ transformer $\mathrm{HV}$ windings using FEA at three locations; top (disk 1), middle (disk 3 ) and bottom (disk 6). The impacts of $1 \%$ to $5 \%$ fault levels on the conventional FRA signature reveal the impediments and barriers of the current FRA practice in identifying incipient fault location and quantifying their levels. The proposed polar plot signatures for the investigated fault levels and locations are obtained and manipulated using the developed DIP code to calculate the three metrics; CBD, RMS and IED as given in Table 4. Similar to the previous case study, Table 4 shows that the three metrics are also increasing with the increase in fault level. On the other hand, Fig. 7 (d-f) reveals that the three metrics are increasing when the fault moves toward the bottom of the HV winding. This may be attributed to the fact that, $\mathrm{HV}$ winding radial deformation is mainly affecting the shunt capacitive components between the deformed location and the core / tank [28]. As can be noticed from the physical dimensions of the two investigated transformers (Fig. 2), the distance between the bottom of the HV winding and the bottom yoke of the transformer core is smaller than that of the top side and hence the radial deformation at the bottom section of the winding will have more effect on the FRA signature than the top section.
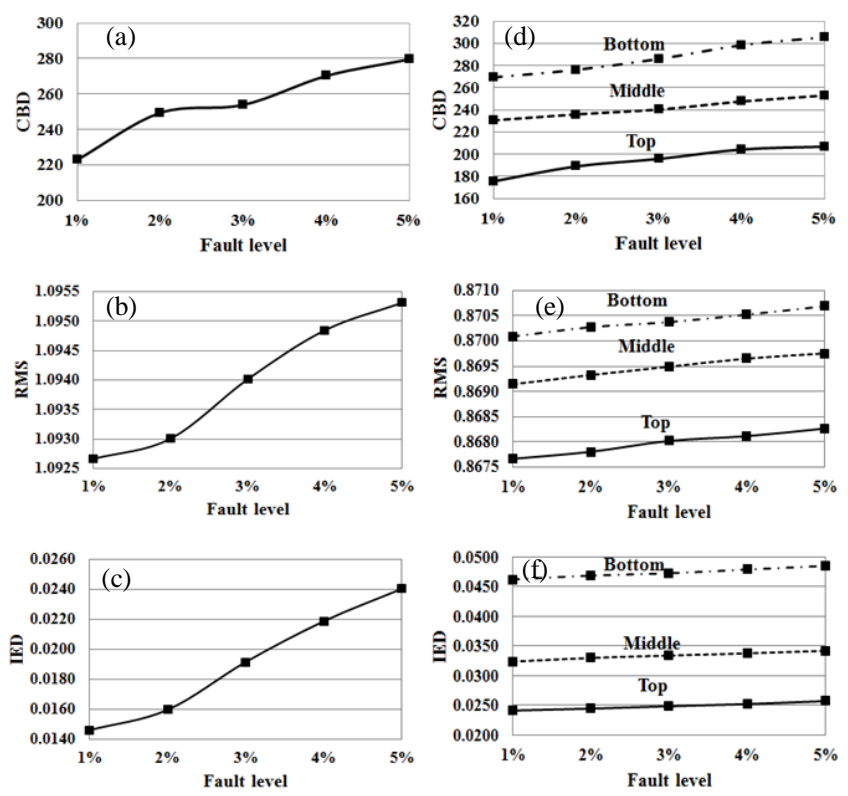

Fig. 7. CBD, RMS and IED trend for the 10kVA transformer radial buckling

Table 4 CBD, RMS and IED metrics of 10kVA transformer $\mathrm{HV}$ winding free buckling

\begin{tabular}{c|c|c|c|c|c|c}
\hline Location & \multicolumn{7}{c}{ Top disk } \\
\hline Metric & $1 \%$ & $2 \%$ & $3 \%$ & $4 \%$ & $5 \%$ & $40 \%$ \\
\hline CBD & 175.35 & 188.92 & 195.65 & 204.17 & 206.69 & 225.02 \\
\hline RMS & 0.867664 & 0.867804 & 0.868018 & 0.868114 & 0.868265 & 0.868428 \\
\hline IED & 0.024130 & 0.024453 & 0.024860 & 0.025220 & 0.025718 & 0.026645 \\
\hline Location & \multicolumn{7}{|c|}{ Middle disk } \\
\hline CBD & 230.42 & 235.86 & 240.21 & 247.52 & 252.87 & 261.69 \\
\hline RMS & 0.869144 & 0.869327 & 0.869492 & 0.869653 & 0.869756 & 0.869806 \\
\hline IED & 0.032377 & 0.033010 & 0.033398 & 0.033774 & 0.034196 & 0.034605 \\
\hline Location & \multicolumn{7}{|c|}{ Bottom disk } \\
\hline CBD & 269.26 & 276.06 & 285.84 & 298.32 & 305.45 & 317.69 \\
\hline RMS & 0.870091 & 0.870277 & 0.870377 & 0.870526 & 0.870692 & 0.870765 \\
\hline IED & 0.046231 & 0.046895 & 0.047286 & 0.047905 & 0.048521 & 0.049063 \\
\hline
\end{tabular}

\subsection{Case study 2: 40MVA transformer}

This case study is conducted to examine the influence of transformer size on the proposed DIP-based polar plot technique; same case studies conducted on the 10kVA transformer are repeated on the 40MVA transformer.

\subsubsection{Forced radial buckling}

Forced radial buckling is simulated on the 40MVA transformer LV winding (phase-A) with fault levels $1 \%$ to $5 \%$ along with $40 \%$. As previously shown, conventional FRA signature won't be able to detect fault levels less than $5 \%$. The polar plots of all investigated fault levels are obtained 
and analysed using the developed DIP code to extract the 15 proposed image features and calculate the three metrics as shown in Table 5 and Fig. 8 (a-c). Results show that, the three metrics are increasing with the increase in fault level with values higher than those obtained for the 10kVA transformer

Table 5 CBD, RMS, IED metrics of 40MVA transformer $\mathrm{LV}$ winding forced buckling

\begin{tabular}{c|c|c|c|c|c|c}
\hline Metric & $1 \%$ & $2 \%$ & $3 \%$ & $4 \%$ & $5 \%$ & $40 \%$ \\
\hline CBD & 1820.93 & 1837.01 & 1850.71 & 1868.48 & 1887.86 & 2557.45 \\
\hline RMS & 2.416197 & 2.416745 & 2.417445 & 2.418095 & 2.418698 & 2.419927 \\
\hline IED & 0.355873 & 0.35875 & 0.36214 & 0.36584 & 0.36964 & 0.393519 \\
\hline
\end{tabular}
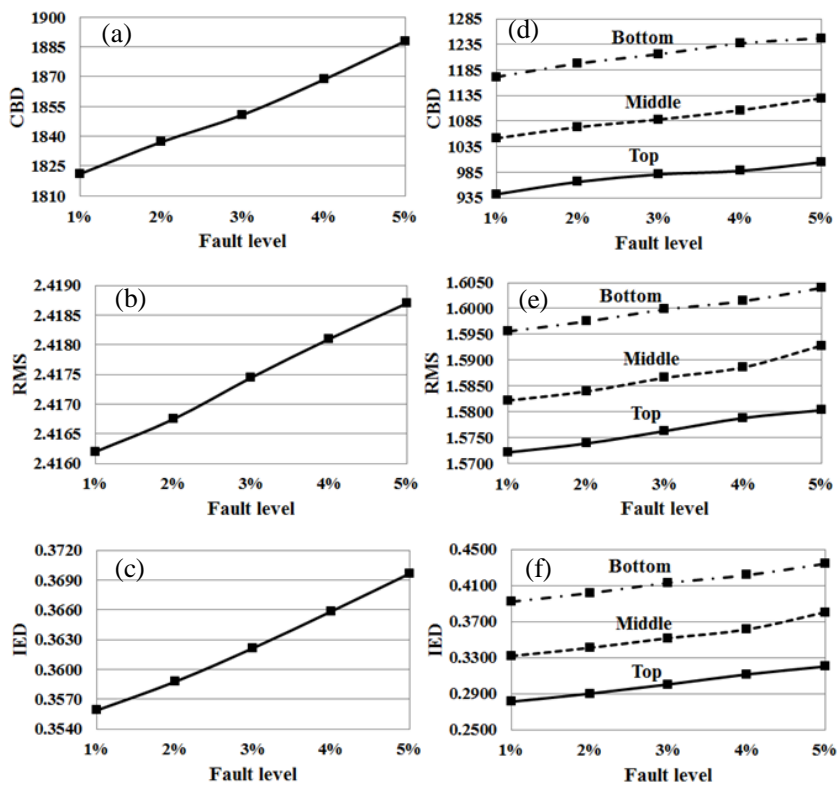

Fig. 8. CBD, RMS and IED trend for the 4OMVA transformer radial buckling

\subsubsection{Free radial buckling}

Various free radial buckling levels are simulated on the top (disk 1), middle (disk 5) and bottom (disk 10) of the 40MVA HV winding. The polar plot of each fault level and location is obtained and the 15 image features are extracted to calculate the proposed three metrics as listed in Table 6 and plotted in Fig. 8 (d-f). As can be seen, the 3 metrics are increasing with the increase in fault level and they are increasing when the fault moves toward the bottom of the windings; same trend was obtained for the 10kVA transformer.

Table 6 CBD, RMS and IED metrics of 40MVA transformer HV winding free buckling

\begin{tabular}{c|c|c|c|c|c|c}
\hline Location & \multicolumn{7}{c}{ Top disk } \\
\hline Metric & $1 \%$ & $2 \%$ & $3 \%$ & $4 \%$ & $5 \%$ & $40 \%$ \\
\hline CBD & 941.84 & 965.97 & 980.73 & 987.52 & 1004.69 & 1344.49 \\
\hline RMS & 1.57215 & 1.573873 & 1.576275 & 1.578795 & 1.580303 & 1.619634 \\
\hline IED & 0.281049 & 0.28993 & 0.300165 & 0.311271 & 0.320055 & 0.488266 \\
\hline Location & \multicolumn{7}{|c}{ Middle disk } \\
\hline CBD & 1051.39 & 1073 & 1088.05 & 1106.13 & 1129.6 & 1599.49 \\
\hline RMS & 1.582195 & 1.583944 & 1.586586 & 1.5886 & 1.592824 & 1.624762 \\
\hline IED & 0.331635 & 0.340728 & 0.351494 & 0.361095 & 0.380253 & 0.510495 \\
\hline Location & \multicolumn{7}{|c|}{ Bottom disk } \\
\hline CBD & 1171.3 & 1197.43 & 1215.81 & 1236.76 & 1246.95 & 1737.26 \\
\hline RMS & 1.595576 & 1.597488 & 1.599812 & 1.601428 & 1.603986 & 1.628125 \\
\hline IED & 0.391897 & 0.401446 & 0.412775 & 0.421305 & 0.434006 & 0.52456 \\
\hline
\end{tabular}

\section{Experimental validation}

Experimental measurements have been conducted on a single phase $2 \mathrm{kVA}, 250 / 120$ volts, dry type transformer. Physical radial buckling with levels of $3 \%$ and $8 \%$ have been staged on the transformer external winding. A frequency response analyser is used to measure the transformer FRA signature with the healthy and deformed winding. Also, FEA is used to simulate a 3D-model of the investigated transformer to compare simulation and practical results. Fig. 9 shows good agreement between the FRA signatures for the healthy and deformed winding when obtained through FEA simulation and practical measurements. Results in Fig. 9 also show the difficulty of identifying the fault using conventional FRA signature. The polar plot signatures are obtained and processed using the proposed DIP code. The 15 features are used to calculate the three proposed metrics for both simulation and practical results as listed in Table 7. Similar to above case studies, the three metrics are increasing with the increase in radial buckling level. It can also be observed that experimental and simulation analysis results have almost similar metrics values which reveal the feasibility of the proposed approach.

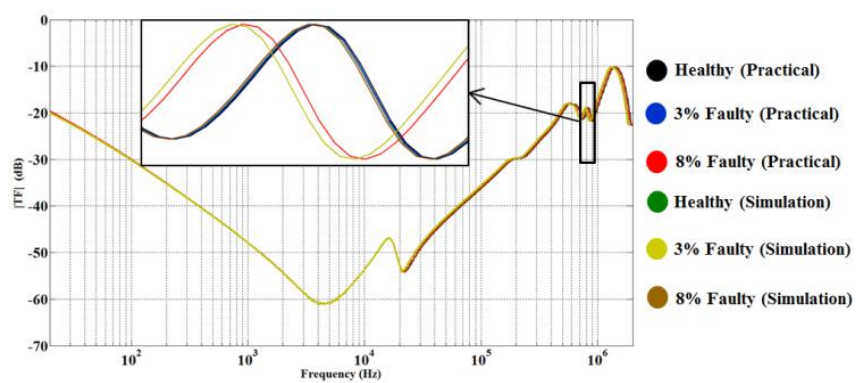

Fig. 9. Conventional FRA signature using practical measurement and simulation analysis for the $2 \mathrm{kVA}$ transformer

Table 7 CBD, RMS and IED metrics of 2kVA transformer LV winding radial buckling

\begin{tabular}{c|c|c|c|c}
\hline Location & \multicolumn{2}{|c|}{ Practical } & \multicolumn{2}{c}{ Simulation } \\
\hline Metric & $3 \%$ & $8 \%$ & $3 \%$ & $8 \%$ \\
\hline CBD & 10.66 & 111.90 & 11.12 & 110.84 \\
\hline RMS & 1.623367 & 1.625126 & 1.638873 & 1.640953 \\
\hline IED & 0.012973 & 0.024648 & 0.011719 & 0.025816 \\
\hline
\end{tabular}

\section{Comparison with numerical techniques}

As previously mentioned, a few studies introduced some numerical metrics such as CC and ASLE calculated from the FRA magnitude signature to ease the FRA interpretation process. These indicators are not widely accepted as reliable tools to analyse the FRA signature as reported in several publications in the literature [8-12]. In order to justify this claim, the magnitude of the FRA signatures for forced buckling levels within the 10kVA transformer is used to calculate the CC and ASLE over a frequency range $10 \mathrm{~Hz}$ to $1 \mathrm{MHz}$ at step of $100 \mathrm{~Hz}$ as per (3) and (4) below.

$$
\begin{gathered}
\mathrm{CC}=\frac{\sum_{=1}^{n} X_{i} Y_{i}}{\sqrt{\sum_{i=1}^{n}\left[X_{i}\right]^{2} \sum_{i=1}^{n}\left[Y_{i}\right]^{2}}} \\
\mathrm{ASLE}=\frac{\sum_{=1}^{n} Y_{i}-X_{i}}{n}
\end{gathered}
$$


where $n$ represents the total number of points of the FRA magnitude data, $X_{i}$ and $Y_{i}$ are the $i^{\text {th }}$ elements of the reference and measured signatures; respectively.

Table 8 shows these metrics along with the three DIPbased classification metrics proposed in this paper. Results show the inconstant trend for the CC. For example, a fault level of $5 \%$ results in a higher correlation between the faulty and healthy signatures than that of the $2 \%$ fault level. On the other hand, ASLE that should be zero in case of perfect correlated signatures is not consistent with the fault level either. For instance a fault level of $4 \%$ leads to ASLE of 0.069 while a fault level of $1 \%$ leads to $1.302 \%$.

Table 8 Statistical and DIP-based metrics for forced radial buckling fault (10kVA transformer)

\begin{tabular}{c|c|c|c|c|c}
\hline $\begin{array}{l}\text { Fault } \\
\text { level }\end{array}$ & CC & ASLE & CBD & RMS & IED \\
\hline $1 \%$ & 0.621 & 1.302 & 222.8 & 1.092663 & 0.014615 \\
$2 \%$ & 0.057 & 0.012 & 249.29 & 1.093005 & 0.015973 \\
$3 \%$ & 0.914 & 0.856 & 253.82 & 1.094014 & 0.019156 \\
$4 \%$ & 0.088 & 0.069 & 270.14 & 1.094845 & 0.021878 \\
$5 \%$ & 0.696 & 1.192 & 279.48 & 1.095310 & 0.024046 \\
\hline
\end{tabular}

This finding aligns well with the results reported in the literature that conclude the imprecision of statistical-based metrics in the FRA signature analysis [11-13]. As shown in Table 8, the proposed DIP-based metrics reveal consistent trend with the fault level which facilitates the identification and quantification of transformer winding radial deformations.

\section{Comparison with other fault types}

To show the suitability of the proposed technique to identify and quantify various winding deformation types, results obtained for transformer winding radial deformation presented in this paper are compared with the results obtained for other winding fault types such as short circuit turns, axial displacement and disk space variation presented in the literature [14-16] in which the same investigated transformers were used.

Table 9 presents the fault threshold levels for various winding faults when applied to the LV and HV windings of the $10 \mathrm{kVA}$ and 40 MVA transformers. As shown in the table, there is no overlapping between threshold bands of the three metrics within each fault type. As few metrics of some faults can probably overlapped, one should look at the three combined metrics to correctly identify and quantify various faults. In some cases, looking at the individual extracted features (physical dimensions, invariant moments and texture analysis) may be of a great help to distinguish various fault types.

Through identifying the threshold fault limits for a particular transformer; a healthy signature can be reported if and only if all three measured metrics are less than the minimum threshold of minor fault level which is $1 \%$. Minor fault can be reported if at least one metric has a value between the proposed minimum and maximum threshold limits while a major fault is reported if at least one metric violates the maximum threshold level.
Table 9 Threshold levels for minor faults within the LV and $\mathrm{HV}$ windings of the 10kVA and 40MVA transformers

\begin{tabular}{|c|c|c|c|c|c|c|c|}
\hline \multirow{2}{*}{\multicolumn{2}{|c|}{ Fault location }} & \multicolumn{6}{|c|}{ Short circuit turns faults } \\
\hline & & \multicolumn{2}{|c|}{ CBD } & \multicolumn{2}{|c|}{ RMS } & \multicolumn{2}{|c|}{ IED } \\
\hline & & Min & Max & Min & Max & Min & Max \\
\hline \multirow{4}{*}{$\frac{\sqrt{2}}{0}$} & LV & 7.76 & 154.23 & 1.096004 & 1.097390 & 0.025059 & 0.040158 \\
\hline & HV/Top & 5.59 & 39.19 & 0.872592 & 0.873590 & 0.048710 & 0.055358 \\
\hline & HV/Middle & 52.67 & 90.78 & 0.874080 & 0.875160 & 0.059371 & 0.067228 \\
\hline & HV/Bottom & 96.05 & 162.36 & 0.875582 & 0.875160 & 0.070316 & 0.078710 \\
\hline \multirow{4}{*}{$\sum_{\stackrel{f}{F}}^{\mathbb{S}}$} & LV & 11.53 & 82.44 & 2.395078 & 2.409629 & 0.225213 & 0.292433 \\
\hline & HV/Top & 120.10 & 191.43 & 1.538625 & 1.539514 & 0.011122 & 0.020432 \\
\hline & HV/Middle & 459.94 & 552.53 & 1.542315 & 1.543488 & 0.048348 & 0.055787 \\
\hline & HV/Bottom & 691.21 & 776.56 & 1.565419 & 1.097390 & 0.152844 & 0.158306 \\
\hline & & \multicolumn{6}{|c|}{ Axial Displacement } \\
\hline & & \multicolumn{2}{|c|}{ CBD } & \multicolumn{2}{|c|}{ RMS } & \multicolumn{2}{|c|}{ IED } \\
\hline & & Min & Max & Min & Max & Min & Max \\
\hline \multirow{2}{*}{$\frac{\overleftrightarrow{s}}{\varrho}$} & LV & 188.94 & 326.71 & 1.083577 & 1.073861 & 0.060020 & 0.117762 \\
\hline & $\mathrm{HV}$ & 80.06 & 139.77 & 0.848081 & 0.845222 & 0.058820 & 0.073257 \\
\hline \multirow{2}{*}{ 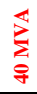 } & LV & 1176.95 & 1324.24 & 2.340710 & 2.339454 & 1.534702 & 1.533434 \\
\hline & $\mathrm{HV}$ & 1296.79 & 1872.78 & 0.032238 & 0.057177 & 0.042565 & 0.059580 \\
\hline & & \multicolumn{6}{|c|}{ Disk Space Variation } \\
\hline & & \multicolumn{2}{|c|}{ CBD } & \multicolumn{2}{|c|}{ RMS } & \multicolumn{2}{|c|}{ IED } \\
\hline & & Min & Max & Min & Max & Min & Max \\
\hline \multirow{3}{*}{$\frac{\sqrt{3}}{\varrho}$} & HV/Top & 7.83 & 16.13 & 0.863410 & 0.863081 & 0.001631 & 0.004133 \\
\hline & HV/Middle & 20.30 & 27.64 & 0.862852 & 0.862438 & 0.005696 & 0.008311 \\
\hline & HV/Bottom & 29.78 & 38.15 & 0.862181 & 0.861758 & 0.009898 & 0.014516 \\
\hline \multirow{3}{*}{ 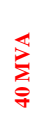 } & HV/Top & 13.37 & 29.06 & 1.538193 & 1.537999 & 0.003231 & 0.004326 \\
\hline & HV/Middle & 33.82 & 53.09 & 1.537820 & 1.537635 & 0.005492 & 0.007080 \\
\hline & HV/Bottom & 63.86 & 81.73 & 1.537483 & 1.537298 & 0.008360 & 0.009920 \\
\hline & & \multicolumn{6}{|c|}{ Radial buckling } \\
\hline & & \multicolumn{2}{|c|}{ CBD } & \multicolumn{2}{|c|}{ RMS } & \multicolumn{2}{|c|}{ IED } \\
\hline & & Min & Max & Min & Max & Min & Max \\
\hline \multirow{4}{*}{$\frac{\mathbb{3}}{\varrho}$} & LV & 222.80 & 617.47 & 1.092663 & 1.103955 & 0.014615 & 0.026640 \\
\hline & HV/Top & 175.35 & 206.69 & 0.867664 & 0.868265 & 0.024130 & 0.025718 \\
\hline & HV/Middle & 230.42 & 252.87 & 0.869144 & 0.869756 & 0.032377 & 0.034196 \\
\hline & HV/Bottom & 269.26 & 305.45 & 0.870091 & 0.870692 & 0.046231 & 0.048521 \\
\hline \multirow{4}{*}{ 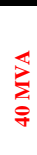 } & LV & 1820.93 & 1887.86 & 2.416197 & 2.418698 & 0.355873 & 0.369640 \\
\hline & HV/Top & 941.84 & 1004.69 & 1.572150 & 1.580303 & 0.281049 & 0.320055 \\
\hline & HV/Middle & 1051.39 & 1129.60 & 1.582195 & 1.592824 & 0.331635 & 0.380253 \\
\hline & HV/Bottom & 1171.30 & 1246.95 & 1.595576 & 1.603986 & 0.391897 & 0.434006 \\
\hline
\end{tabular}

\section{Conclusion}

Current FRA interpretation technique mainly relies on personal expertise which may result in inconsistent reports for the same FRA signature. Moreover, it is extremely hard to identify and quantify incipient mechanical deformations of low fault levels using the conventional FRA interpretation technique. This paper investigates the ability of polar plot and digital image processing techniques to identify and quantify transformer winding radial deformations. The DIP techniques are employed to extract the various polar plot image features including geometric, invariant moments and texture analysis features. The extracted features are used to calculate three unique classification parameters namely; city-block distance, root mean square and image Euclidean distance. The proposed approach is successfully used to identify and quantify radial 
deformation within the windings of two power transformers. Feasibility of the proposed approach has been validated through experimental measurements. The threshold levels of the proposed three classification metrics obtained in this paper are compared with those obtained for other winding fault types applied for the same investigated transformers. Results show that the proposed technique can be successfully employed to detect various transformer winding deformations. The proposed technique is easy to implement within current frequency response analysers to systematize the FRA interpretation process.

\section{References}

[1] A. J. Kachler and I. Hohlein, "Aging of cellulose at transformer service temperatures. Part 1: Influence of type of oil and air on the degree of polymerization of pressboard, dissolved gases, and furanic compounds in oil," Electrical Insulation Magazine, IEEE, vol. 21, pp. 15-21, 2005.

[2] A. Abu-Siada, "Correlation of furan concentration and spectral response of transformer oil-using expert systems," Science, Measurement \& Technology, IET, vol. 5, pp. 183-188, 2011.

[3] A. Abu-Siada and S. Islam, "A Novel Online Technique to Detect Power Transformer Winding Faults," Power Delivery, IEEE Transactions on, vol. 27, pp. 849-857, 2012.

[4] N. Abeywickrama, Y. V. Serdyuk, and S. M. Gubanski, "Effect of Core Magnetization on Frequency Response Analysis (FRA) of Power Transformers," Power Delivery, IEEE Transactions on, vol. 23, pp. 1432-1438, 2008.

[5] S. D. Mitchell and J. S. Welsh, "Modeling Power Transformers to Support the Interpretation of Frequency-Response Analysis," Power Delivery, IEEE Transactions on, vol. 26, pp. 2705-2717, 2011.

[6] J. C. Gonzales Arispe and E. E. Mombello, "Detection of Failures Within Transformers by FRA Using Multiresolution Decomposition," Power Delivery, IEEE Transactions on, vol. 29, pp. 1127-1137, 2014.

[7] CIGER, "Mechanical-condition assessment of transformer windings using Frequency Response Analysis (FRA)," in Tutorial of Cigre WG A2.26 Convener, ed. Patrick Picher, Canada: CIGER, 2007, p. 21.

[8] V. Behjat and M. Mahvi, "Statistical approach for interpretation of power transformers frequency response analysis results," IET Science, Measurement \& Technology, vol. 9, pp. 367-375, 2015.

[9] S. A. Ryder, "Methods for comparing frequency response analysis measurements," in Electrical Insulation, 2002. Conference Record of the 2002 IEEE International Symposium on, 2002, pp. 187-190.

[10]K. Jong-Wook, P. ByungKoo, J. Seung Cheol, K. Sang Woo, and P. PooGyeon, "Fault diagnosis of a power transformer using an improved frequency-response analysis," IEEE Transactions on Power Delivery, vol. 20, pp. 169-178, 2005.

[11]V. Behjat, M. Mahvi, and E. Rahimpour, "New statistical approach to interpret power transformer frequency response analysis: non-parametric statistical methods," IET Science, Measurement \& Technology, vol. 10, pp. 364-369, 2016.

[12]K. P. Badgujar, M. Maoyafikuddin, and S. V. Kulkarni, "Alternative statistical techniques for aiding SFRA diagnostics in transformers," IET Generation, Transmission \& Distribution, vol. 6, pp. 189-198, 2012.

[13]M. F. M. Yousof, C. Ekanayake, and T. K. Saha, "Frequency response analysis to investigate deformation of transformer winding," IEEE Transactions on Dielectrics and Electrical Insulation, vol. 22, pp. 2359-2367, 2015.

[14] O. Aljohani, A. Abu-Siada, "Application of Digital Image Processing to Detect Short Circuit Turns in Power Transformers using Frequency Response Analysis", IEEE
Transactions on Industrial Informatics, Vol. 12, No. 6, pp. 2062-2073, December 2016.

[15] O. Aljohani, A. Abu-Siada, "Application of DIP to Detect Power Transformers Axial Displacement and Disk Space Variation using FRA Polar Plot Signature", IEEE Transactions on Industrial Informatics, Vol. 13, Issue 4, pp. 1794-1805, February 2017.

[16] O. Aljohani, A. Abu-Siada, "Application of Digital Image Processing to Detect Transformer Bushing Faults and Oil Degradation using FRA Polar Plot Signature", IEEE Transactions on Dielectrics and Electrical Insulation, Vol. 24, Issue 1, pp. 428-436, August 2017

[17]A. Hyun-Mo, L. Ji-Yeon, K. Joong-Kyoung, O. Yeon-Ho, J. Sang-Yong, and H. Sung-Chin, "Finite-Element Analysis of Short-Circuit Electromagnetic Force in Power Transformer," Industry Applications, IEEE Transactions on, vol. 47, pp. 1267-1272, 2011.

[18]H. Wang and K. L. Butler, "Finite element analysis of internal winding faults in distribution transformers," Power Delivery, IEEE Transactions on, vol. 16, pp. 422-428, 2001.

[19]N. Hashemnia, A. Abu-Siada, and S. Islam, "Improved power transformer winding fault detection using FRA diagnostics part 1: axial displacement simulation," Dielectrics and Electrical Insulation, IEEE Transactions on, vol. 22, pp. 556-563, 2015.

[20]N. Hashemnia, A. Abu-Siada, and S. Islam, "Improved power transformer winding fault detection using FRA diagnostics part 2: radial deformation simulation," Dielectrics and Electrical Insulation, IEEE Transactions on, vol. 22, pp. 564-570, 2015.

[21]R. C. Gonzalez, Rafael C. Gonzalez, Richard E. Woods, Digital image processing, Upper Saddle River, Pearson/Prentice Hall, 2008.

[22] A. McAndrew, "An introduction to digital image processing with matlab notes for SCM2511 image processing," School of Computer Science and Mathematics, Victoria University of Technology, pp. 1-264, 2004.

[23]D. Xiaolong and S. Khorram, "A feature-based image registration algorithm using improved chain-code representation combined with invariant moments," Geoscience and Remote Sensing, IEEE Transactions on, vol. 37, pp. 23512362, 1999.

[24] G. Plonka and M. Jianwei, "Nonlinear Regularized Reaction-Diffusion Filters for Denoising of Images With Textures," Image Processing, IEEE Transactions on, vol. 17, pp. 1283-1294, 2008.

[25] W. Pedrycz, A. Amato, V. Di Lecce, and V. Piuri, "Fuzzy Clustering With Partial Supervision in Organization and Classification of Digital Images," Fuzzy Systems, IEEE Transactions on, vol. 16, pp. 1008-1026, 2008.

[26] S. Bing and F. Jufu, "A Fast Algorithm for Image Euclidean Distance," in Pattern Recognition, 2008. CCPR '08. Chinese Conference on, 2008, pp. 1-5.

[27]L. Yi-Ming, C. Li-Fang, L. Yuan, and W. Hao-Tian, "An Image Matching Algorithm Based on SIFT and Improved LTP," in Computational Intelligence and Security (CIS), 2013 9th International Conference on, 2013, pp. 432-436.

[28] A. Abu-Siada, N. Hashemnia, S. Islam, and M.A.S. Masoum, "Understanding Power Transformer Frequency Response Analysis Signatures", IEEE Electrical Insulation Magazine, Vol. 29, No. 3, pp. 48-56, May-June 2013. 